\title{
TREATMENT DURATION FOR ABSENCE EPILEPSY
}

The effects of 6 months of treatment followed by a 5- to 6-week withdrawal period of ethosuximide (ESM) in 3 children, ages 5, 10, and 10 years, with new onset absence epilepsy are reported from the Departments of Neurology, State University of New York at Stony Brook, and the Division of Child Neurology, Schneider Children's Hospital and Albert Einstein College of Medicine, New York. The patients had responded promptly to ESM at levels of $75-90 \mathrm{mcg} / \mathrm{ml}$, and their 24-hour ambulatory EEGs were normal. After this early withdrawal of ESM, 2 patients remained seizure-free and their 24-hour ambulatory EEG was burst free, and one was seizure-free but showed $3 / \mathrm{sec}$ spike-wave bursts in the EEG. Optimizing medication until both clinical and electrographic seizure activity are continuously suppressed, as determined by 24-hour ambulatory EEG, allows early withdrawal of treatment without increased risk of relapse. (Amit R, Vitale S, Maytal J. How long to treat childhood onset absence epilepsy. Clin Elecroencephalogr July 1995;26:163165). (Reprints: Rami Amit MD, Neurology, HSC 12-020, SUNY at Stony Brook, Stony Brook, NY 11794).

COMMENT. In this study, ethosuximide was chosen as the drug of first choice in the treatment of childhood onset absence epilepsy. In my experience, while ethosuximide and valproate are equally effective in controlling clinical absence attacks, valproate appears, in uncontrolled studies, to be superior in the suppression of electrographic $3 / \mathrm{sec}$ spikewave discharges. A comparative trial of these drugs using 24-hour ambulatory EEG monitoring would be of interest. The authors found that a burst-free routine EEG will not guarantee a complete remission of electrographic seizure activity, and a clean 24-hour ambulatory EEG is essential before attempting this recommended early drug withdrawal.

A 2 year seizure free interval is the generally recommended time before antiepileptic drug withdrawal. (Shinnar S, Zacharowicz L, Moshe SL. Initiation and cessation of antiepileptic drug therapy in children and adolescents. Acta Neuropediatr 1995;1(3):153-166).

\section{SERUM LIPIDS AND LONG-TERM ANTIEPILEPTIC THERAPY}

Serum levels of total cholesterol (TC), high-density lipoprotein cholesterol (HDL-C), low-density lipoprotein cholesterol (LDL-C), very LDL-C, and triglycerides (TGs) were determined in 119 children with epilepsy treated with AEDs for 7 months to 10 years (mean, 5.8 years) and in 125 healthy controls at the Hospital General de Galicia, Clinico Universitario, Santiago de Compostela, Spain. In patients receiving carbamazepine (58) or phenobarbital (22), mean TC, HDL-C, and LDL-C levels were significantly higher than in the control group. Serum TC exceeded the accepted safe level of $200 \mathrm{mg} / \mathrm{dl}$ in $41 \%$ of carbamazepine-treated children and in 50\% of those receiving phenobarbital, compared to only $12 \%$ of controls. In those on valproic acid (39), mean TC and LDL-C levels and mean TC/HDL-C and LDL-C/HDL-C ratios were significantly lower than controls. (Eiris JM et al. Effects of long-term treatment with antiepileptic drugs on serum lipid levels in children with epilepsy. Neurology June 1995;45:1155-1157). (Reprints: Dr M Castr-Gago, Department of Pediatrics, Division of Pediatric Neurology, Hospital General de Galicia, 15705 Santiago de Compostela, Spain).

COMMENT. Serum cholesterol levels should be carefully monitored in children treated with carbamazepine or phenobarbital for epilepsy. 